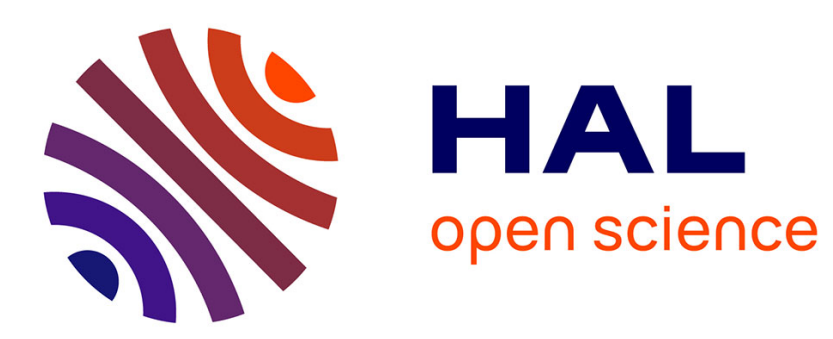

\title{
Optiques X-UV multicouches et techniques annexes
}

\author{
P. Dhez
}

\section{To cite this version:}

P. Dhez. Optiques X-UV multicouches et techniques annexes. Revue de Physique Appliquée, 1988, 23 (10), pp.1577-1577. 10.1051/rphysap:0198800230100157700 . jpa-00245987

\section{HAL Id: jpa-00245987 https://hal.science/jpa-00245987}

Submitted on 1 Jan 1988

HAL is a multi-disciplinary open access archive for the deposit and dissemination of scientific research documents, whether they are published or not. The documents may come from teaching and research institutions in France or abroad, or from public or private research centers.
L'archive ouverte pluridisciplinaire HAL, est destinée au dépôt et à la diffusion de documents scientifiques de niveau recherche, publiés ou non, émanant des établissements d'enseignement et de recherche français ou étrangers, des laboratoires publics ou privés. 


\section{REVUE DE PHYSIQUE APPLIQUÉE}

\section{Optiques X-UV multicouches et techniques annexes}

\section{Introduction}

Les premiers miroirs interférentiels adaptés aux rayons $\mathrm{X}$ furent réalisées en 1954, mais leur application à des optiques ne date que de quelques années. Les progrès décisifs pour leur optimisation sont dus à E. Spiller qui vers 1982 fut le premier à démontrer les possibilités réelles de ces miroirs et comment ils permettaient de s'affranchir des contraintes imposées jusqu'à cette époque en X-UV par les optiques sous incidence rasante. Très récemment, des optiques dites de «Bragg-Fresnel " ont été développées en combinant les propriétés de diffraction des réseaux et celles des réflecteurs de Bragg (V. V. Aristov et al.). Les articles sélectionnés pour ce numéro spécial s'efforcent de montrer l'état actuel des réalisations en optique X-UV, en particulier en astrophysique (R. C. Catura et al.) et dans les expériences en vue d'obtenir la fusion à l'aide de lasers pulsés (R. Benattar, L. Knight et al.).

L'article d'introduction (J. M. André and B. Pardo) est un rappel du problème général de l'interaction d'une onde électromagnétique et d'un milieu stratifié. Il montre qu'étant donné les constantes optiques disponibles en X-UV, le traitement mathématique de ce problème se pose d'une façon particulière et en quoi il diffère de celui étudié depuis longtemps pour le domaine visible et les ondes radar. Pour obtenir des réflectivités notables à différentes longueurs d'ondes, on est conduit à considérer des systèmes périodiques constitués de couple de métaux de numéro atomique très différents (A. Rosenbluth) et à étudier la formation des interfaces de ces matériaux (C. Piecuch). Pour améliorer la qualité des interfaces on s'efforce de suivre leur formation in-situ en suivant la variation de réflectivité X (J. P. Chauvineau) ou par interférométrie en visible ( $\mathrm{P}$. Houdy). Les études a posteriori sont des mesures de réflectivité en $\mathrm{X}$ ou $\mathrm{X}$-UV réalisées aux longueurs d'ondes d'utilisation. Elles permettent de prendre en compte différents types d'imperfections dont les effets sont difficiles à modéliser dans les calculs (R. Barchewitz et al., F. Christensen, L. Nevot, E. Spiller).

Les performances de ces optiques interférentielles sont étroitement liées à l'obtention de couches périodiques ultraminces, de un à quelques nanomètres d'épaisseur. Les problèmes technologiques posés par la préparation de ces couches ultraminces rejoignent ceux rencontrés de plus en plus fréquemment dans la fabrication des circuits à très haut degré d'intégration (VLSI) et en optoélectronique. La réalisation des optiques interférentielles X-UV nécessitant la préparation de milieux stratifiés artificiellement à l'échelle quasiatomique est entrepris activement dans plusieurs pays en liaison avec des projets de microtechnologie. Par exemple au Japon, cet enjeu technologique est intégré dans le « Nano Project »; un article décrit l'état des réalisations et des projets dans ce pays ( $T$. Namioka).

D'un point de vue plus général, on peut remarquer que l'étude des défauts de surfaces, des matériaux artificiels à très faible période et de la diffusion aux interfaces, sont une classe de problèmes apparaissant de plus en plus fréquemment dans divers domaines, aussi bien en physique, qu'en physico chimie ou en biologie. La série d'articles présentée ici montre comment, à sa façon, la réalisation et le test d'optiques interférentielles X-UV contribue à une meilleure connaissance de cette classe de problèmes.

P. Dhez

Editeur Invité

Laboratoire pour l'Utilisation du Rayonnement Electromagnétique

Bât. 209D, Centre Universitaire Paris-Sud

91405 Orsay Cedex 